\title{
PLAGES DE LA DISCORDE
}

Jennifer Bidet, Elsa Devienne

Le Seuil | «Actes de la recherche en sciences sociales »

$2017 / 3 \mathrm{~N}^{\circ} 218$ | pages 4 à 9

ISSN 0335-5322

ISBN 9782021340587

Article disponible en ligne à l'adresse :

http://www.cairn.info/revue-actes-de-la-recherche-en-sciencessociales-2017-3-page-4.htm

\section{Pour citer cet article :}

Jennifer Bidet, Elsa Devienne« Plages de la discorde », Actes de la recherche en sciences sociales $2017 / 3$ ( $\left.\mathrm{N}^{\circ} 218\right)$, p. 4-9.

DOI 10.3917/arss.218.0004

Distribution électronique Cairn.info pour Le Seuil.

(C) Le Seuil. Tous droits réservés pour tous pays.

La reproduction ou représentation de cet article, notamment par photocopie, n'est autorisée que dans les limites des conditions générales d'utilisation du site ou, le cas échéant, des conditions générales de la licence souscrite par votre établissement. Toute autre reproduction ou représentation, en tout ou partie, sous quelque forme et de quelque manière que ce soit, est interdite sauf accord préalable et écrit de l'éditeur, en dehors des cas prévus par la législation en vigueur en France. Il est précisé que son stockage dans une base de données est également interdit. 
$-$

NORI AUX

TOURISTES... 


\section{Jennifer Bidet et Elsa Devienne}

\section{Plages de la discorde}

"L'accès aux plages et à la baignade est interdit à compter de la signature du présent arrêté jusqu'au 31 août 2016 à toute personne n'ayant pas une tenue correcte, respectueuse des bonnes mœurs et de la laïcité. »

Arrêté municipal, Ville de Cannes, 28 juillet 2016.

Été 2016 : les arrêtés « anti-burkini » émis par des municipalités du Sud-Est de la France alimentent la chronique médiatique. L'état d'urgence, le terrorisme et la laïcité, alors omniprésents dans le débat public français, s'invitent dans un espace pensé comme éloigné des préoccupations du quotidien. Ces arrêtés ont pour but d'empêcher les femmes portant un maillot de bain couvrant leur torse et leur tête de venir sur la plage, au motif d'éviter les troubles à l'ordre public qu'une telle tenue pourrait susciter après l'attentat de Nice du 14 juillet 2016. Lété précédent, c'est le séjour à Vallauris (Alpes-Maritimes) du roi d'Arabie Saoudite qui défraye la chronique. La privatisation temporaire d'une plage publique par un personnage cumulant pouvoir politique et économique touche à un symbole de la démocratisation de l'accès aux loisirs depuis l'avènement des congés payés. Ces événements sont venus rappeler que le littoral, et a fortiori les plages de sable qui en sont l'élément le plus convoité, constitue un enjeu de luttes politiques et économiques.

Cette politisation des plages n'est pas un phénomène inédit, ni spécifiquement français. La montée du niveau des mers, sous l'effet du changement climatique et de l'urbanisation, ou encore la privatisation croissante des côtes constituent des préoccupations publiques majeures. En outre, la construction des plages comme espaces de loisirs s'est faite historiquement dans un rapport de concurrence avec d'autres fonctions et par l'appropriation de cet espace par un groupe au détriment d'autres ${ }^{1}$. Plus qu'une simple réponse à l'actualité, ce dossier a été pensé en réaction à la manière dont la plage a été étudiée par les sciences sociales. Selon un récit aujourd'hui bien établi, la plage

1. Johan Vincent, L'Intrusion balnéaire : les populations littorales bretonnes et vendéennes face au tourisme, 1800-1945, Rennes, PUR, 2007 ; Connie Y. Chiang, Shaping the Shoreline. Fisheries and Tourism on the Monterey Coast, Seattle, University of Washington Press, 2008. 
a longtemps été vue comme un univers hostile à l'activité humaine, avant de faire l'objet d'une mutation du regard dans le monde occidental au cours des XVIII et XIX ${ }^{\mathrm{e}}$ siècles, pour enfin devenir un espace désiré et le support privilégié d'un temps libéré des contraintes sociales, le temps des vacances ${ }^{2}$. Ce récit, aujourd'hui débattu, a eu tendance à arrêter l'histoire des plages à la fin du XIX ${ }^{\mathrm{e}}$ siècle et à surestimer la linéarité du processus de diffusion des usages ludiques des rivages. Le regard des sciences sociales sur la plage s'est ensuite focalisé de manière excessive sur l'affaiblissement des contraintes sociales et des rapports de domination sur le sable. Enfin, cet espace a souvent été étudié comme un isolat dans des approches invisibilisant les contextes socio-politiques et territoriaux dans lesquels il s'inscrit. Autant d'écueils que ce dossier cherche à éviter en pensant d'abord les plages comme des territoires contestés.

Ainsi, une nouvelle histoire sociale des plages a remis en cause l'approche diffusionniste et linéaire du développement des pratiques balnéaires longtemps privilégiée par l'histoire culturelle. Christophe Granger a révèlé les rapports sociaux qui président à l'imposition du bronzage et de la semi-nudité par des classes moyennes en ascension sociale contre les élites traditionnelles dans la France de l'entre-deuxguerres $^{3}$. Pour Andrew Kahrl, les petits propriétaires noirs du Sud des États-Unis ont été les premiers à être dépossédés de leurs terrains au gré de la massification du tourisme et du développement d'un secteur immobilier de luxe en bord de mer ${ }^{4}$. Les travaux réunis dans ce dossier montrent que l'histoire des luttes pour l'appropriation des plages continue au XX siècle - autour de la surveillance des comportements des baigneurs de classes populaires et des minorités ethniques et raciales dans le contexte étasunien ${ }^{5}$ ou, dans le contexte brésilien, autour du déclin des plantations sucrières, de l'arrivée de surfeurs issus de milieux citadins aisés et de la reconversion des descendants de planteurs dans le tourisme ${ }^{6}$. Contre une vision linéaire de la balnéarisation des littoraux, ce dossier souligne également la diversité des formes de mises en tourisme - de l'hôtel all-inclusive au petit établissement "rustique »en fonction des groupes sociaux présents sur le territoire et des conflits que cette mise en tourisme occasionne, révèle ou amplifie.

Nombre de travaux sur les pratiques vacancières ont insisté sur leur fonction émancipatrice qui, en autorisant une distance aux rôles de la vie quotidienne, estomperait les différences sociales et favoriserait l'expression de l'individualité 7 . Emblème de la conquête des vacances comme «triomphe d'une utopie ${ }^{8}$, la plage est alors présentée comme un espace hétérotopique détaché des pesanteurs sociales ${ }^{9}$.

2. Alain Corbin, Le Territoire du Harvard University Press, 2012. vide. L'Occident et le désir du rivage, 1750-1840, Paris, Aubier, 1998.

3. Christophe Granger, Les Corps d'été. Naissance d'une variation saisonnière, $X X^{e}$ siècle, Paris, Autrement, 2012.

4. Andrew W. Kahrl, The Land Was Ours. African American Beaches from Jim Crow to the Sunbelt South, Cambridge (Mass.),
5. Voir dans ce numéro, Elsa Devienne, " "Il y a des lois sur la plage!" Régulation et surveillance des comportements sur les plages de Los Angeles, années 19101970 ", Actes de la recherche en sciences sociales, 218, 2017, p. 10-25.

6. Voir dans ce numéro, Tristan Loloum, «Derrière la plage, les plantations.
Touristification du littoral et recomposition des élites dans le Nordeste brésilien ", Actes de la recherche en sciences sociales, 218, 2017, p. 46-63.

7. Jean Viard, Penser les vacances, La Tour-d'Aigues, Éd. de l'Aube, 2007 ; Jean-Didier Urbain, Sur la plage : mœurs et coutumes balnéaires (XIXe-XXe siècles), Paris, Payot, 1994
8. Jean Viard, Le Triomphe d'une utopie. Vacances, loisirs, voyages la révolution des temps libres, La Tour-d'Aigues, Éd. de l'Aube, 2015.

9. Jean Rieucau et Jérôme Lageiste, "La plage, un territoire singulier : entre hétérotopie et antimonde ", Géographie et cultures, 67, 2008, p. 3-6. 


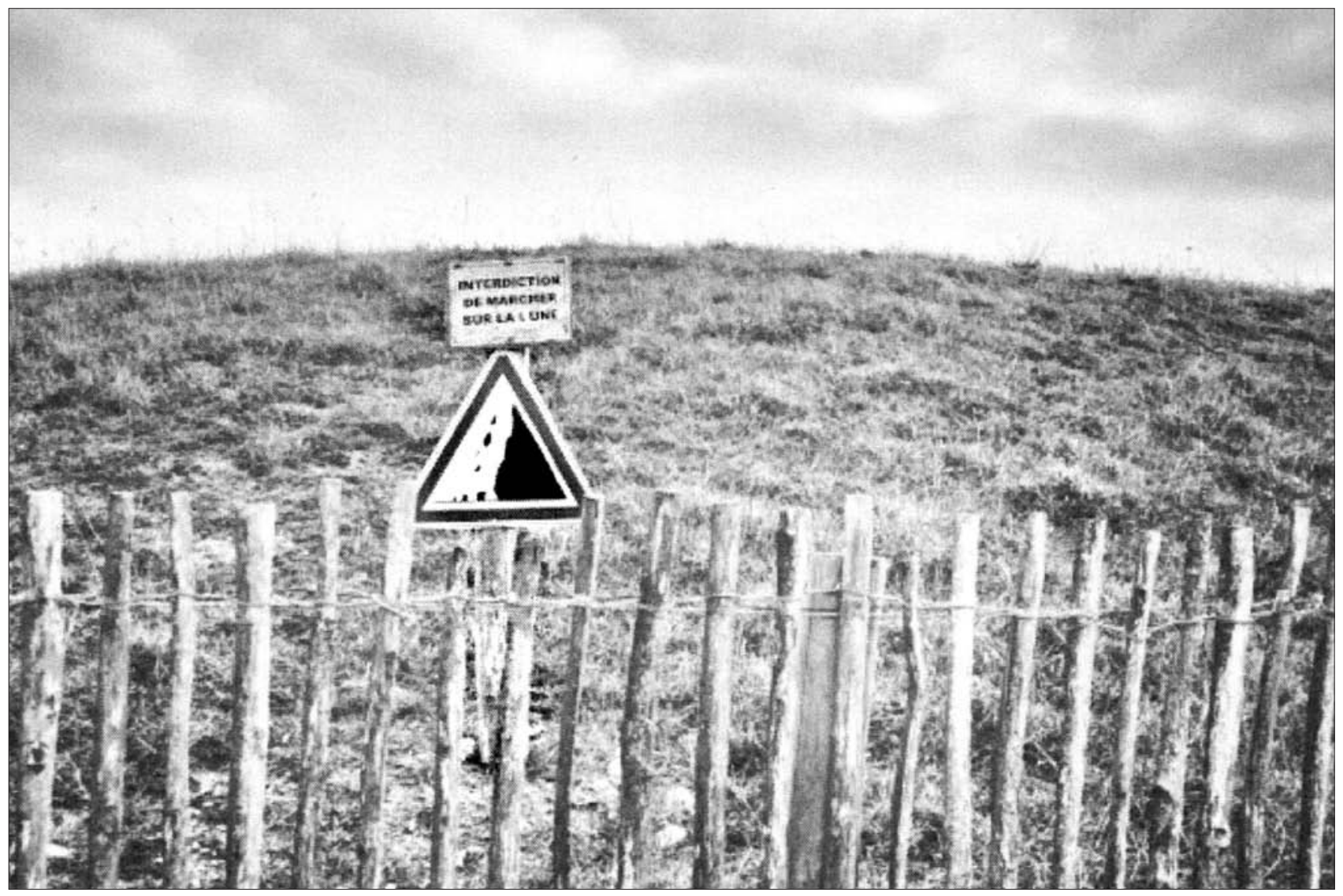

LA FRAGILITÉ ENVIRONNEMENTALE des littoraux est à la fois le résultat de l'exploitation humaine de la nature et, bien souvent, un argument en faveur d'une appropriation privée de certains espaces littoraux au nom de la préservation de l'environnement. Cette dune, située dans le Finistère, a été reconstruite artificiellement afin de protéger des maisons construites sous le niveau de la mer. 
Des travaux rappellent toutefois le maintien de fortes inégalités dans l'accès au départ en vacances ${ }^{10}$. En faisant de l'enchantement touristique non pas un résultat mais un objet de l'analyse, ils mettent également en lumière les mécanismes d'euphémisation des rapports marchands dans la relation de service du secteur touristique ${ }^{11}$. La plage peut alors être saisie comme une scène révélatrice des phénomènes de distinction ou de concurrence entre groupes sociaux ${ }^{12}$. L'instauration d'une barrière économique à l'entrée d'une plage, les normes vestimentaires imposées aux femmes et aux hommes ou encore l'instauration d'une séparation raciale dans l'organisation du littora ${ }^{13}$ sont autant de réalités qui soulignent le maintien des rapports de domination dans ces espaces. Dans le contexte algérien, les plages payantes constituent un lieu de rencontre privilégié entre des groupes sociaux - des familles algériennes de classes supérieures et des jeunes Français de milieu populaire issus de l'immigration algérienne - qui n'ont que peu d'occasions de se croiser dans d'autres contextes. Ces complexes balnéaires révèlent l'existence d'un espace social transnational où le passé colonial et les hiérarchies sociales propres aux deux pays se combinent pour générer des formes de classement inédites autour de l'usage de catégories ethno-nationales ${ }^{14}$. L'espace de la plage offre aussi des ressources pour contester une hiérarchie sociale existante, du moins la modifier. À Zanzibar, la plage est propice à la construction de relations intimes entre des hommes tanzaniens, appelés beach boys, et des touristes occidentales car elle met en scène des corps sexués. Au sein des unions qui peuvent en découler, les capitaux économiques et culturels se combinent à la possession de compétences locales spécifiques pour nourrir des trajectoires d'ascension sociale inédites ${ }^{15}$.

Enfin, l'espace de la plage a souvent été traité comme un isolat géographique et social. Si l'analyse de la pratique des seins nus par Jean-Claude Kauffman éclaire l'évolution des formes d'auto-contrôle et des rapports de $\operatorname{sex}^{16}$, la plage y est réduite à son rôle de support de pratiques ludiques rendant possible le dénudement, partiel et contrôlé. Ce dossier propose au contraire de réinscrire la plage dans son contexte spatial plus large. À l'échelle urbaine d'abord, car c'est souvent dans le cadre d'une agglomération que se déterminent les aménagements, les publics et les usages admis sur les littoraux. L'échelle régionale doit aussi être mobilisée dans la mesure où la mise en tourisme du littoral est parfois liée à l'érosion d'une activité économique dominante dans l'arrière-pays. La dimension internationale, enfin, doit être prise en compte. En effet, l'abaissement des coûts du trafic aérien dans les années 1970 a entraîné l'essor

10. Louis Chauvel, "Du pain et des vacances : la consommation des catégories socioprofessionnelles s'homogénéise-t-elle (encore)? ", Revue française de sociologie, 40(1), 1999, p. 79-96 ; Céline Rouquette, "Départs en vacances : la persistance des inégalités ", Économie et statistique, 345, 2001, p. 33-53.

11. Bertrand Réau et Franck Poupeau, "L'enchantement du monde touristique ",
Actes de la recherche en sciences sociales, 170, 2007, p. 4-13.

12. Patrick Champagne, "Les paysans à la plage ", Actes de la recherche en sciences sociales, 1(2), 1975, p. 21-24 ; Christophe Andréo, "Surveillance et contrôle des jeunes des quartiers populaires sur une plage marseillaise à la fin des années 1990 ", Genèses, 67, 2007, p. 89-108.

13. A. W. Kahrl, op. cit. ; Sophie Chevalier,
"Comment partager les mêmes espaces? Les classes moyennes à Durban (Afrique du Sud) ", Espaces et sociétés, 148-149, 2012, p. 129-144.

14. Voir dans ce numéro, Jennifer Bidet, "Blédards" et "immigrés" sur les plages algériennes. Luttes de classement dans un espace social transnational ", Actes de la recherche en sciences sociales, 218 , 2017, p. 64-81.
15. Voir dans ce numéro, Altaïr Despres, "Des histoires avec lendemains. Intimité transnationale et ascension sociale des beach boys de Zanzibar ", Actes de la recherche en sciences sociales, 218 , 2017, p. 82-99.

16. Jean-Claude Kauffman, Corps de femmes, regards d'hommes. Sociologie des seins nus, Paris, Nathan, 1995. 
du tourisme balnéaire dans les pays en développement et permis la mise en contact de populations différenciées - à l'image des rencontres étudiées à Zanzibar ou sur les côtes algériennes. C'est aussi à l'articulation de ces différentes échelles que l'espace du littoral est constitué en enjeu de luttes : le statut particulier octroyé à la plage de Pampelonne à Saint-Tropez (par un amendement à la loi littoral régulant la présence d'établissements commerciaux sur le rivage) a été l'objet de mobilisations contradictoires entre élus municipaux et nationaux, hauts-fonctionnaires et associations locales $^{17}$. En recontextualisant ainsi l'objet " plage », ce dossier éclaire les logiques qui président à la balnéarisation différentielle des littoraux et à leur appropriation par des groupes sociaux antagonistes ou conjoncturellement alliés. La plage devient alors bien plus que le simple support d'une reconfiguration de l'économie locale et d'une suspension des rapports sociaux : elle apparait comme un enjeu de luttes au sein de circuits politiques et économiques complexes et, en cela, contribue à la production de rapports sociaux de classe, de sexe et de race ${ }^{18}$ inédits. 